\title{
On the induction schema for decidable predicates
}

\author{
Lev D. Beklemishev * \\ Steklov Mathematical Institute \\ Gubkina 8, 117966 Moscow, Russia \\ e-mail: bekl@mi.ras.ru
}

\begin{abstract}
We study the fragment of Peano arithmetic formalizing the induction principle for the class of decidable predicates, $I \Delta_{1}$. We show that $I \Delta_{1}$ is independent from the set of all true arithmetical $\Pi_{2}$-sentences. Moreover, we establish the connections between this theory and some classes of oracle computable functions with restrictions on the allowed number of queries. We also establish some conservation and independence results for parameter free and inference rule forms of $\Delta_{1}$-induction.

An open problem formulated by J. Paris (see $[4,5])$ is whether $I \Delta_{1}$ proves the corresponding least element principle for decidable predicates, $L \Delta_{1}$ (or, equivalently, the $\Sigma_{1}$-collection principle $B \Sigma_{1}$ ). We reduce this question to a purely computation-theoretic one.
\end{abstract}

\section{Introduction and motivation}

The schema of induction for decidable predicates $I \Delta_{1}$ is considered to be rather exotic. Indeed, the stronger schema of induction for r.e. predicates $I \Sigma_{1}$ appears more naturally in the formalization of various mathematical and metamathematical arguments and presently has the status of one of the most important and well-understood fragments of PA.

Nonetheless, the class of decidable relations is natural from a recursiontheoretic point of view, and we shall see below that $I \Delta_{1}$ also corresponds to sufficiently interesting models of computation. The present paper is an attempt to fill several obvious gaps in our understanding of $I \Delta_{1}$.

In Section 2 we accumulate some basic information on $\Delta_{1}$-induction. In Section 3 we prove that $I \Delta_{1}$ is independent from the set of all true arithmetical $\Pi_{2}$-sentences. Some corollaries on the parameter-free versions of $I \Delta_{1}$ are obtained in Section 4. Our further analysis of $I \Delta_{1}$ is based on a reduction of $I \Delta_{1}$ to an inference rule form of $\Delta_{1}$-induction, which is obtained in Section 5 . In particular, this allows us to characterize in Section 6 the $\Sigma_{3}$-theorems of $I \Delta_{1}$.

In Section 7 we approach the problem formulated by J. Paris, that is, the question whether $I \Delta_{1}$ contains the $\Sigma_{1}$-collection schema $B \Sigma_{1}$. We only obtain partial results in this direction. However, a characterization of the classes of provably total computable functions of the extensions of $I \Delta_{1}$ by true $\Pi_{2}$-axioms is obtained, which yields a reduction of $I \Delta_{1}$ versus $B \Sigma_{1}$ problem to a purely computation-theoretic question.

\footnotetext{
` Supported by Lise Meitner Research Fellowship, RFBR and INTAS.
} 


\section{Preliminaries}

Elementary Arithmetic EA is a first order theory formulated in the language with the symbols $\left(0,1,+, \cdot, 2^{x}, \leq,=\right)$ which all have the usual meaning in the structure of natural numbers $\mathbb{N}$. EA has some finite set of basic open axioms defining these symbols together with the induction schema for bounded formulas (this theory is denoted $I \Delta_{0}^{\exp }(\exp )$ in [5]). It is known that EA is finitely axiomatizable.

The schema $I \Delta_{1}$ is defined as follows.

$$
I \Delta_{1}: \quad \forall x(\varphi(x) \leftrightarrow \psi(x)) \rightarrow I_{\varphi},
$$

for all $\varphi \in \Sigma_{1}, \psi \in \Pi_{1}$, where

$$
I_{\varphi}:=\varphi(0) \wedge \forall x(\varphi(x) \rightarrow \varphi(x+1)) \rightarrow \forall x \varphi(x)
$$

is the usual induction formula for $\varphi$ (parameters in $\varphi$ and $\psi$ are allowed). Abusing the terminology, $I \Delta_{1}$ will also denote the extension of EA by this schema.

This definition is based on the characterization of decidable predicates as those which are simultaneously $\Sigma_{1}$ and $\Pi_{1}$. Alternatively, one can define decidable predicates as those having computable characteristic functions. A universal Turing machine can be naturally represented in EA, that is, one can write out a $\Sigma_{1}$-formula $\Phi_{e}(x) \simeq y$ expressing " $e$-th computable function on input $x$ converges to $y$ ". Then one arrives at the following axiom $a I \Delta_{1}$ :

$$
\forall e\left(\forall x \exists y \leq 1 \Phi_{e}(x) \simeq y \rightarrow I_{\Phi_{e}(x) \simeq 0}\right) .
$$

The proof of the following lemma is straightforward, so we omit it.

Lemma 1. I $\Delta_{1}$ and aI $\Delta_{1}$ are equivalent over $E A$.

This presents an evidence that our formulation of $I \Delta_{1}$ is reasonably stable - different approaches yield equivalent systems. We also have the following

Corollary 1. I $\Delta_{1}$ is finitely axiomatizable (over EA).

On a par with $I \Delta_{1}$ we shall consider the least element principle and the order induction schema. Letting

$$
\begin{aligned}
L_{\varphi} & :=\exists x \varphi(x) \rightarrow \exists x(\varphi(x) \wedge \forall y<x \neg \varphi(y)), \\
O I_{\varphi} & :=\forall x(\forall y<x \varphi(y) \rightarrow \varphi(x)) \rightarrow \forall x \varphi(x),
\end{aligned}
$$

the schemata $L \Delta_{1}$ and $O I \Delta_{1}$ are naturally defined as follows:

$$
\begin{aligned}
L \Delta_{1}: & \forall x(\varphi(x) \leftrightarrow \psi(x)) \rightarrow L_{\varphi}, \\
O I \Delta_{1}: & \forall x(\varphi(x) \leftrightarrow \psi(x)) \rightarrow O I_{\varphi},
\end{aligned}
$$

where $\varphi \in \Sigma_{1}$ and $\psi \in \Pi_{1}$.

Obviously, over EA one has $L_{\varphi} \Leftrightarrow O I_{\neg \varphi} \Rightarrow I_{\neg \varphi}$, hence $L \Delta_{1} \vdash I \Delta_{1}$, but the converse is an open problem formulated by J. Paris (see $[5,4]$ ). 
Open problem: $I \Delta_{1} \vdash L \Delta_{1}$ ?

Why does the usual argument for the equivalence of $L$ and $I$ not work? Usually one proves

$$
I_{\forall y \leq x \varphi(y)} \Rightarrow O I_{\varphi}
$$

which is also provable in EA, but EA need not recognize that $\forall y \leq x \varphi(y)$ is decidable $\left(\Delta_{1}\right)$, if so is $\varphi$. The natural principle establishing this property is the schema of $\Sigma_{1}$-collection:

$$
B \Sigma_{1}: \quad \forall x \leq a \exists y \varphi(x, y) \rightarrow \exists z \forall x \leq a \exists y \leq z \varphi(x, y),
$$

for $\varphi \in \Sigma_{1}$. Informally, this axiom states that if a computable function is total on an interval $[0, a]$, then there is a uniform bound on the size of its values on $[0, a]$. R. Gandy (see [5]) proved that $B \Sigma_{1}$ is equivalent to $L \Delta_{1}$, hence the usual argument does not help to solve Paris' problem.

$B \Sigma_{1}$ is a well-studied fragment of arithmetic. It is known that $B \Sigma_{1}$ follows from $\Sigma_{1}$-induction $I \Sigma_{1}$, but not conversely. In fact, by J. Paris [7] and $\mathrm{H}$. Friedman, $B \Sigma_{1}$ is $\Pi_{2}$-conservative over EA. Yet, $B \Sigma_{1}$ is strong from the point of view of the set of all true $\Pi_{2}$-sentences, $T h_{\Pi_{2}}(\mathbb{N})$. C. Parsons [9] showed that $B \Sigma_{1}$ is independent from this (non-r.e.) theory: $T h_{\Pi_{2}}(\mathbb{N}) \nvdash B \Sigma_{1}$. So, we can draw the following diagram of fragments of arithmetic below $I \Sigma_{1}$ :

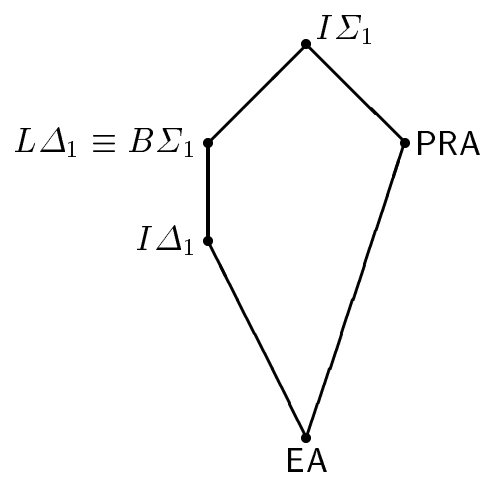

Here, PRA denotes the fragment of the primitive recursive arithmetic in the language of EA, which can be axiomatized over EA by infinitely many $\Pi_{2}$-axioms stating the totality of the generating functions of the Grzegorczyk hierarchy. ${ }^{1}$ Notice that PRA is not included in $B \Sigma_{1}$ by J. Paris and H. Friedman, and $B \Sigma_{1}$ is not included in PRA by C. Parsons. It is also well-known that $I \Sigma_{1}$ is $\Pi_{2^{-}}$ conservative over PRA [8]. It is open, whether $I \Delta_{1}$ is properly included in $B \Sigma_{1}$ (we conjecture that it is).

$\overline{1}$ Alternatively, it can be axiomatized over EA by the inference rule form of $\Sigma_{1}$ induction. 
In the next section we shall show that $I \Delta_{1}$ properly extends EA. In fact, we shall improve a result of $\mathrm{C}$. Parsons and show that $T h_{\Pi_{2}}(\mathbb{N}) \nvdash I \Delta_{1}$. This also implies that $I \Delta_{1}$ is not included in PRA.

\section{Independence of $\Delta_{1}$-induction}

Our goal in this section is to prove the following result.

Theorem 1. $T h_{\Pi_{2}}(\mathbb{N}) \nvdash I \Delta_{1}$.

Proof. We shall first prove the simpler statement EA $\nvdash I \Delta_{1}$. Then we shall modify this proof to obtain the stronger statement of the theorem. The idea of the proof is a variation of the one in [2].

Since $I \Delta_{1}$ is $\Pi_{2}$-conservative over EA, it would be hopeless to try to prove the consistency of EA in $I \Delta_{1}$. So, rather than showing EA $\nvdash I \Delta_{1}$ directly we shall construct a $\Pi_{2}$-sentence $\pi$ such that it is easy to prove

$$
\mathrm{EA}+\pi \nvdash I \Delta_{1}+\pi
$$

In fact, for a suitable $\pi$ we want

$$
\mathcal{F}(\mathrm{EA}+\pi) \neq \mathcal{F}\left(I \Delta_{1}+\pi\right),
$$

where $\mathcal{F}(T)$ is the class of provably total computable functions of a theory $T$.

Recall that a function $g \in \mathcal{F}(T)$ iff for some $\varphi(x, y) \in \Sigma_{1}$,

1. $g(\boldsymbol{x})=y \Leftrightarrow \mathbb{N} \vDash \varphi(\boldsymbol{x}, y)$,

2. $T \vdash \forall \boldsymbol{x} \exists y \varphi(\boldsymbol{x}, y)$.

It is known that $\mathcal{F}(\mathrm{EA})$ coincides with the class of (Kalmar) elementary functions $\mathcal{E}$. These can also be characterized in the following ways:

1. The functions whose computation time is bounded by $\exp ^{(n)}(x)$ for some constant $n$, where

$$
\exp (x)=2^{x}, \exp ^{(n+1)}(x)=\exp \left(\exp ^{(n)}(x)\right)
$$

2. The closure of $0,1,+, \cdot$, exp, projection functions and the characteristic function of $\leq$ by composition and bounded recursion.

3. Bounded recursion in 2. can be replaced (modulo adding some simple initial functions) by either of the following operators: bounded $\mu$-operator, bounded sum, bounded product.

For an arbitrary $T$ containing EA not too much can be said about $\mathcal{F}(T)$ except that $\mathcal{F}(T)$ contains $\mathcal{E}$ and is closed under composition. It is also easy to see that 1$) \mathcal{F}(T)$ only depends on the $\Pi_{2}$-fragment of $T$; 2) adding true $\Pi_{1}$-axioms to $T$ does not change the class $\mathcal{F}(T)$.

For a class of functions $K$ let $\mathbf{E}(K)$ denote the closure of $\mathcal{E} \cup K$ under composition and bounded recursion. $\mathbf{C}(K)$ is the closure of $\mathcal{E} \cup K$ under composition alone; we also write $\mathbf{C}(f)$ instead of $\mathbf{C}(\{f\})$. 
The classes $\mathbf{C}(f)$ precisely correspond to the provably total computable functions of true $\Pi_{2}$-sentences. To see this let $\mathrm{EA}(f)$ denote a conservative extension of EA formulated in the language with the symbols for all elementary functions and a new function symbol $f$. Axioms of $\operatorname{EA}(f)$ are (open) defining equations for all elementary functions and the schema of induction for open formulas not containing $f$. In other words, no mathematical axioms about $f$ are added. Any bounded formula not containing $f$ is $\mathrm{EA}(f)$-equivalent to an open one. Moreover, it is known that $\mathrm{EA}(f)$ admits a purely universal axiomatization.

Lemma 2. If $\pi$ is a sentence in the language of $E A$ of the form $\forall x \exists y \varphi(x, y)$ with $\varphi$ bounded, then $\mathcal{F}(E A+\pi)=\mathbf{C}(f)$, where $f(x):=\mu y . \varphi(x, y)$.

Proof. Representing $\varphi$ as an open formula and adding the axiom $\forall x \varphi(x, f(x))$ to $\mathrm{EA}(f)$ we obtain a conservative purely universal extension of $\mathrm{EA}+\pi$. Then the inclusion $\mathcal{F}(\mathrm{EA}+\pi) \subseteq \mathbf{C}(f)$ follows from Herbrand's theorem. The opposite inclusion is immediate (details can be found, e.g., in [1]).

Now we are going to analyze the classes $\mathcal{F}\left(I \Delta_{1}+\pi\right)$ for $\pi \in \Pi_{2}$. By the abuse of notation, the set of all open formulas in the language of $\operatorname{EA}(f)$ (which may involve $f$ ) will be denoted $\mathbf{C}(f)$. This notation is justified, because every open formula in $\operatorname{EA}(f)$ is equivalent to the one of the form $t(\boldsymbol{x})=0$, for a suitable $\mathbf{C}(f)$-term $t$. I $\mathbf{C}(f)$ denotes the induction schema for open formulas and the corresponding extension of $\mathrm{EA}(f)$.

Let $\pi=\forall x \exists y \varphi(x, y)$ be as in Lemma 2 with $\varphi$ bounded. The bounded formula $\varphi(x, y) \wedge \forall z<y \neg \varphi(x, z)$ represents the graph of the function $f(x):=\mu y \cdot \varphi(x, y)$ and hence defines a canonical interpretation of $f$ in $\mathrm{EA}+\pi$. For this interpretation we observe the following

Lemma 3. $I \Delta_{1}+\pi$ interprets $I \mathbf{C}(f)$.

Proof. It is sufficient to show that the interpretation of any open formula of $\mathrm{EA}(f)$ is provably $\Delta_{1}$ in $\mathrm{EA}+\pi$, that is, it is provably equivalent both to a $\Sigma_{1}$ and to a $\Pi_{1}$-formula.

First, we notice that for any $\operatorname{EA}(f)$ term $t(\boldsymbol{x})$, the formula $t(\boldsymbol{x})=y$ is $\operatorname{EA}(f)$ equivalent to a purely existential formula $\psi_{0}(\boldsymbol{x}, y)$ as well as to a purely universal formula $\psi_{1}(\boldsymbol{x}, y)$ such that every atomic subformula of $\psi_{i}$ either does not contain the symbol $f$ or has the form $f(u)=v$ for $u$ and $v$ variables. This is the usual process of unwinding complex terms, which works in pure logic with equality. For example,

$$
\begin{aligned}
f(h(f(x), a))=y & \leftrightarrow \exists u \exists v(f(x)=u \wedge h(u, a)=v \wedge f(v)=y) \\
& \leftrightarrow \forall u \forall v(f(x)=u \wedge h(u, a)=v \rightarrow f(v)=y),
\end{aligned}
$$

which has the required form assuming $h$ is an elementary function.

Since every open formula is equivalent to the one of the form $t(\boldsymbol{x})=0$, it follows, more generally, that any open formula has such purely existential and purely universal representations in $\operatorname{EA}(f)$. 
Under the considered interpretation atomic subformulas of the form $f(u)=v$ are replaced in $\psi_{0}$ and $\psi_{1}$ by the bounded formulas

$$
\varphi(u, v) \wedge \forall z<v \neg \varphi(u, z),
$$

which yields, respectively, $\Sigma_{1^{-}}$and $\Pi_{1}$-formulas in the language of EA. Since $\mathrm{EA}+\pi$ proves all the interpreted theorems of $\mathrm{EA}(f)$, the interpretations of $\mathbf{C}(f)$ formulas are provably $\Delta_{1}$ in EA $+\pi$.

It is useful at this point to introduce a dual form of induction, which I call the interval search principle:

$$
S_{\varphi}:=\varphi(a) \wedge \neg \varphi(b) \wedge a<b \rightarrow \exists x \in[a, b)(\varphi(x) \wedge \neg \varphi(x+1)) .
$$

The search principle essentially states that a $0-1$ valued function $f$ (represented by $\varphi$ on $[a, b]$ ) such that $f(a)=0$ and $f(b)=1$ must change its value at some point $x$ of the interval, that is, $f(x)=0$ and $f(x+1)=1$. Notice that we do not claim that the point $x$ should be the minimal possible one.

Trivially, over EA, $S_{\neg \varphi} \Rightarrow I_{\varphi}$ (take $a=0$ ), and $I_{\neg \varphi(x+a)} \Rightarrow S_{\varphi}$. It follows that $S \Delta_{1} \equiv I \Delta_{1}$ and $S \mathbf{C}(f) \equiv I \mathbf{C}(f){ }^{2}$

Now we prove that $I \mathbf{C}(f)$ verifies that $f$ has a local maximum on any bounded interval. Notice that the predicate " $f$ has a local maximum at $x$ " can be expressed by an open formula $\operatorname{LM}(a, b, x)$ :

$$
\begin{aligned}
&(x=a\wedge f(x) \geq f(x+1)) \vee(x=b \wedge f(x) \geq f(x-1)) \vee \\
&(a<x<b \wedge f(x-1) \leq f(x) \wedge f(x+1) \leq f(x)) .
\end{aligned}
$$

Lemma 4. $I \mathbf{C}(f)$ proves that $f$ has a local maximum point on any bounded interval:

$$
\forall a<b \exists x \in[a, b] \operatorname{LM}(a, b, x) .
$$

Proof. Apply $S \mathbf{C}(f)$ to the formula $\varphi(x):=f(x+1) \geq f(x)$ and the interval $[a, b-1]$. If $\neg \varphi(a)$ then the local maximum is at $a$; if $\varphi(b-1)$ then the local maximum is at $b$. Otherwise, by $S \mathbf{C}(f)$ we obtain a point $x$ such that $x+1$ is a local maximum of $f$.

Remark 1. The same argument shows that $I \mathbf{C}(f)$ verifies the existence of a local maximum for any function, whose graph is definable by a $\mathbf{C}(f)$-formula.

Hence, if EA $+\pi \vdash I \Delta_{1}+\pi$, then by Lemma 2 the problem of finding a local maximum point of $f(x)=\mu y \cdot \varphi(x, y)$ on a bounded interval belongs to $\mathbf{C}(f)$. However, by the following lemma this is, generally, impossible.

Lemma 5. There is a recursive function $f$ (with an elementary graph) such that no function $t(a, b) \in \mathbf{C}(f)$ faithfully computes a local maximum point of $f$ on interval $[a, b]$, that is, for every such $t$,

$$
\mathbb{N} \vDash \exists a, b(a<b \wedge \neg L M(a, b, t(a, b))) .
$$

\footnotetext{
${ }^{2}$ Notice that the reduction of $S$ to $I$ depends on the presence of parameters. Below we show that the parameter free versions of $I \Delta_{1}$ and $S \Delta_{1}$ are not equivalent.
} 
Take $\pi:=\forall x \exists y \varphi(x, y)$, where $\varphi$ is a bounded formula representing the graph of $f$ from this lemma. Then, by the construction, $\mathrm{EA}+\pi \nvdash I \Delta_{1}+\pi$, which shows EA $\nvdash I \Delta_{1}$. So, it remains for us to construct such an $f$.

Before doing so we notice that any $\mathbf{C}(f)$-term can be considered as a program with an oracle for a function $f$ which may only ask a bounded number of oracle queries (this number is bounded by the number of occurrences of the symbol $f$ in $t$ and does not depend on the input of the program). The running time is then bounded by an elementary function of the size of the input together with all the oracle answers.

Notice that the naive search algorithm in the worst case uses $b-a+1$ oracle queries to find a local maximum point of $f$ on $[a, b]$. The more efficient binary search algorithm uses $2 \cdot \log (b-a)+O(1)$ queries: first check, if $a$ or $b$ is a local maximum; if not, compare the values of $f$ at $\lfloor(a+b) / 2\rfloor$ and $\lfloor(a+b) / 2\rfloor+1$ to decide, if a local maximum of $f$ is found in the left or in the right half of $[a, b]$. It is intuitively clear that a bounded number of queries in generally not enough to find a local maximum point. In fact, it would be sufficient for our present purposes to prove a simple logarithmic lower bound on the number of queries first. (Sharp upper and lower bounds of the form $\log _{\alpha} n+O(1)$, where $\alpha$ is the 'golden section' constant $\frac{1+\sqrt{5}}{2}$, were subsequently obtained in [10]).

Lemma 6. Fix an interval $[a, b]$ and assume that $M_{f}$ is an oracle Turing machine such that for any function $f, M_{f}(a, b)$ asks $\leq \log (b-a+1)-\log 3$ queries. Then there is a function $f$ such that $M_{f}$ does not faithfully compute a local maximum point of $f$ on $[a, b]$.

Proof. Consider such a machine $M_{f}$. Let $q:=\log (b-a+1)-\log 3$, then the number of points of the interval $[a, b]$ is $b-a+1=3 \cdot 2^{q}$.

Define $f$ on $[a, b]$ simultaneously with the computation of $M_{f}(a, b)$. The computation is devided into stages. Stage $n$ begins when the $n$-th query is being asked. At every stage $n, f$ will be defined on two subintervals $\left[a, a_{n}\right]$ and $\left[b_{n}, b\right]$ of the interval $[a, b]$ and undefined everywhere inbetween. $f$ may be defined arbitrarily outside the interval $[a, b]$.

At stage 0 set $f(a)=f(b):=0, a_{0}:=a, b_{0}:=b$ and start computing $M_{f}(a, b)$.

At stage $n$, as soon as the next value of $f(u)$ for some $u$ is queried, choose one of the three possible cases.

Case 1: $u \in\left(a_{n}, b_{n}\right)$ and $u-a_{n} \leq b_{n}-u$. Set $a_{n+1}:=u, b_{n+1}:=b_{n}$ and define $f(x):=f\left(a_{n}\right)+\left(x-a_{n}\right)$ for all $x \in\left(a_{n}, u\right]$. Output the value $f(u)$ and continue the computation of $M_{f}$ (go to the next stage).

Case 2: $u \in\left(a_{n}, b_{n}\right)$ and $u-a_{n}>b_{n}-u$. Set $b_{n+1}:=u, a_{n+1}:=a_{n}$ and define $f(x):=f\left(b_{n}\right)+\left(b_{n}-x\right)$ for $x \in\left[u, b_{n}\right)$. Output the value $f(u)$ and continue the computation of $M_{f}$.

Case 3: $u \notin\left(a_{n}, b_{n}\right)$. Then $f(u)$ is already defined. Output this value, do not change $a_{n}$ and $b_{n}$, and continue the computation of $M_{f}$.

We claim that after $n$ stages ( $n$ queries), if $n \leq q$, the interval $\left(a_{n}, b_{n}\right)$ will be nonempty. Let $p_{n}$ denote the number of points in $\left[a_{n}, b_{n}\right]$. Recall that $p_{0}=3 \cdot 2^{q}$ 
and by the construction

$$
p_{n+1} \geq \frac{1}{2} p_{n}-\frac{1}{2} .
$$

Summing up the geometric progression we obtain

$$
p_{n}>\frac{1}{2^{n}} p_{0}-1 .
$$

Hence, $p_{n}>3-1=2$, which means that $\left(a_{n}, b_{n}\right)$ is nonempty.

We assume that $M_{f}(a, b)$ terminates after $n \leq q$ queries and outputs a local maximum point $x$ of $f$. By the construction of $f, x$ may only belong to $\left[a_{n}, b_{n}\right]$, because there are no local maxima anywhere else on $[a, b]$. Yet, if $x \in\left[a_{n}, b_{n}\right]$ we can always define $f$ on the remaining nonempty interval $\left(a_{n}, b_{n}\right)$ in such a way that $x$ will not be a local maximum point. A contradiction.

Now we are going to prove Lemma 5 .

Proof. Let $t_{0}(a, b), t_{1}(a, b), \ldots$ be an (effective) enumeration of all $\mathbf{C}(f)$-terms of the variables $a, b$. The required function $f$ will be 'pasted' of finite functions $f_{n}:\left[a_{n}, b_{n}\right] \rightarrow \mathbb{N}$ defined on disjoint intervals and such that $t_{n}\left(a_{n}, b_{n}\right)$ is not a local maximum of $f_{n}$.

Let $q_{n}$ be the number of occurrences of the symbol $f$ in $t_{n}$. Choosing $b_{n}-a_{n} \geq$ $3 \cdot 2^{q_{n}}-1$ and letting $f_{n}$ be as in the previous lemma would imply that $t_{n}$ does not faithfully compute its local maximum on $\left[a_{n}, b_{n}\right]$. It only has to be guaranteed, in addition, that $f$ has an elementary graph and is everywhere defined.

To this end, the whole construction should be made effective. As in [1] we define $f$ in terms of a clock Turing machine, that is, a Turing machine with an additional read-only tape which contains the current time (the number of steps of the computation). The content of the clock tape can be copied on the work tape in one step. Obviously, clock Turing machines are polynomially simulated by the ordinary ones.

The clock Turing machine computing the values of $f$ will be constructed in such a way that, whenever a value $f(x)$ is being defined, it has to exceed the current value of the clock. Since the size of the full protocol of the computation is polynomial in the number of steps, this entails that the graph of $f$ is elementary. This requirement, however, is easy to fulfill by modifying the construction of $f$ in Lemma 6, e.g., as follows.

Assume we have already constructed the functions $f_{i}$ for $i<n$ and now consider the term $t_{n}$. Pick a fresh interval $[a, b]$ of size $3 \cdot 2^{q_{n}}$ such that $a>$ $\max \{x: f(x)$ is defined $\}$. Define $f(x):=$ current time, if $x=b$ or $(x \leq a$ and $f(x)$ is undefined). Start computing the value $t_{n}(a, b)$ in accordance with the construction in Lemma $6\left(t_{n}\right.$ plays the role of $\left.M_{f}\right)$. Instead of defining $f(x):=f\left(a_{n}\right)+\left(x-a_{n}\right)$ in Case 1 , put $f(x):=f\left(a_{n}\right)+T+\left(x-a_{n}\right)$, where $T$ is the current time (and similarly in Case 2). In Case 3 the value $f(u)$ may now not yet be defined (if $u>b$ ); in this case put $f(u):=T$.

After $q_{n}$ stages the term $t_{n}(a, b)$ will be evaluated to a certain $x$. We can define $f$ on the remaining interval $\left(a_{q_{n}}, b_{q_{n}}\right)$ in such a way that $x$ is not a local 
maximum point, if $x \in\left[a_{q_{n}}, b_{q_{n}}\right]$, and the values of $f$ exceed the current value of the clock. Then $f$ satisfies all the requirements. This completes the proof of Lemma 5 and shows the independence of $I \Delta_{1}$ from EA.

We prove Theorem 1 essentially by relativizing the above arguments. Assume $T h_{\Pi_{2}}(\mathbb{N}) \vdash I \Delta_{1}$. Since $I \Delta_{1}$ is finitely axiomatizable, there is a particular true $\Pi_{2}$-sentence $\pi_{0}=\forall x \exists y \psi(x, y)$ such that $\mathrm{EA}+\pi_{0} \vdash I \Delta_{1}$. We know that $\mathcal{F}(\mathrm{EA}+$ $\left.\pi_{0}\right)=\mathbf{C}(g)$, where $g(x):=\mu y \cdot \psi(x, y)$.

Notice that the presence of additional initial recursive functions does not change anything in the proof of Lemma 5, so we obtain the following version.

Lemma 7. Let $g$ be a total recursive function. Then there is a recursive function $f$ (with an elementary graph) such that no function $t(a, b) \in \mathbf{C}(g, f)$ faithfully computes a local maximum point of $f$ on interval $[a, b]$, that is, for every such $t$,

$$
\mathbb{N} \vDash \exists a, b(a<b \wedge \neg L M(a, b, t(a, b))) .
$$

As above, let $\varphi(x, y)$ represent the graph of $f$ and let $\pi:=\forall x \exists y \varphi(x, y)$. We obviously have $\mathcal{F}\left(\mathrm{EA}+\pi_{0}+\pi\right)=\mathbf{C}(g, f)$. Hence, open formulas in the language of $\mathrm{EA}(g, f)$ are (interpreted as) provably $\Delta_{1}$ in EA $+\pi_{0}+\pi$. As in Lemma 4 we obtain that $\pi_{0}+\pi+I \Delta_{1}$ interprets $I \mathbf{C}(g, f)$, therefore it proves that on any interval a local maximum point of $f$ exists. Yet, Lemma 7 implies that this is impossible to prove in EA $+\pi_{0}+\pi$, which completes the proof of Theorem 1.

\section{Parameter free schemata}

The results of the previous section can be strengthened and yield additional information about parameter free $\Delta_{1}$-induction schema $I \Delta_{1}^{-}$. We also let $S \Delta_{1}^{-}$ denote the schema $S \Delta_{1}$ without parameters. (In both cases, parameters are the additional free variables occurring in the formulas $\varphi(x)$ and $\psi(x)$.) In general, we think that $S \Delta_{1}^{-}$is more interesting than $I \Delta_{1}^{-}$, for the reasons explained in Section 6 . Here we show that $S \Delta_{1}^{-}$is properly stronger than $I \Delta_{1}^{-}$and collect some other facts on these two schemata.

The proof of Lemmas 3 and 4 directly shows that $\pi+S \Delta_{1}^{-}$proves the existence of a local maximum point of the witnessing function of $\pi$. Hence, as before $\mathrm{EA}+\pi \nvdash S \Delta_{1}^{-}$, for any true $\Pi_{2}$-sentence $\pi$. Also notice that any r.e. set $X$ of true $\Pi_{2}$-sentences follows from a single true $\Pi_{2}$-sentence - the uniform $\Pi_{2}$-reflection principle for $\mathrm{EA}+X$. Thus, we obtain the following corollary.

Corollary 2. For any sound r.e. $\Pi_{2}$-axiomatized theory $T, T \nvdash S \Delta_{1}^{-}$.

This implies, for example, that $S \Delta_{1}^{-}$is independent from PRA, as well as from the set of all $\Pi_{2}$-theorems of PA.

On the other hand, it is easy to see that the schema $S \Delta_{1}^{-}$follows from the set of all true $\Pi_{2}$-sentences.

Lemma 8. $T h_{\Pi_{2}}(\mathbb{N}) \vdash S \Delta_{1}^{-}$. 
Proof. To derive $S \Delta_{1}^{-}$consider the premise $\forall x(\varphi(x) \leftrightarrow \psi(x))$. If it is true, then the conclusion $S_{\varphi}$ is equivalent to a formula of complexity $\Pi_{2}$ and follows from $T h_{\Pi_{2}}(\mathbb{N})$. If it is false, then for some $n$ we have

$$
\mathbb{N} \vDash \neg(\varphi(\bar{n}) \leftrightarrow \psi(\bar{n})) .
$$

This formula is a boolean combination of $\Sigma_{1}$-formulas, so it is provable in $T h_{\Pi_{2}}(\mathbb{N})$, and thus

$$
T h_{\Pi_{2}}(\mathbb{N}) \vdash \neg \forall x(\varphi(x) \leftrightarrow \psi(x)) .
$$

If $S \Delta_{1}^{-}$were finitely axiomatizable, by compactness Lemma 8 would contradict Corollary 2.

Corollary 3. $S \Delta_{1}^{-}$is not finitely axiomatizable.

We can also separate $S \Delta_{1}^{-}$and $I \Delta_{1}^{-}$. First, almost as in the proof of Lemma 8 we obtain

Lemma 9. $T h_{\Pi_{1}}(\mathbb{N}) \vdash I \Delta_{1}^{-}$.

Proof. One only has to realize that a formula of the form $\neg(\varphi(\bar{n}) \leftrightarrow \psi(\bar{n}))$, if valid, is provable already in $T h_{\Pi_{1}}(\mathbb{N})$.

Corollary 4. $I \Delta_{1}^{-} \nvdash S \Delta_{1}^{-}$.

Proof. Notice that since $T h_{\Pi_{1}}(\mathbb{N}) \vdash I \Delta_{1}^{-}$, for any true $\Pi_{2}$-sentence $\pi$, we have

$$
\mathcal{F}\left(\pi+I \Delta_{1}^{-}\right)=\mathcal{F}(\mathrm{EA}+\pi) .
$$

The latter property fails for $S \Delta_{1}^{-}$, as we have seen from the proof of Corollary 2 .

\section{Reduction of $I \Delta_{1}$ to $\Delta_{1}$-induction rule}

In order to approach $I \Delta_{1}$ vs. $B \Sigma_{1}$ question a more careful analysis of the schema $I \Delta_{1}$ is necessary. We simplify the picture by reducing $I \Delta_{1}$ to the corresponding $\Delta_{1}$-induction rule.

$\Delta_{1}$-induction rule is the following rule of inference:

$$
\Delta_{1}-\mathrm{IR}: \quad \frac{\forall x(\varphi(x) \leftrightarrow \psi(x))}{I_{\varphi}},
$$

where $\varphi \in \Sigma_{1}$ and $\psi \in \Pi_{1}$.

Notice that the premise and the conclusion of the rule are (equivalent to) $\Pi_{2}$-sentences, whereas the schema $I \Delta_{1}$ has arithmetical complexity $\Pi_{3}$. Thus, the behaviour of the rule will be easier to describe in computational terms than that of the axiom schema.

Also notice that all the usual theories, such as EA or PRA, are closed under this rule: by Herbrand's theorem provably $\Delta_{1}$-formulas $\varphi$ of EA are provably equivalent to bounded formulas, hence the induction principles $I_{\varphi}$ for such $\varphi$ are all EA-provable. On the other hand, the results of the previous section show that there is a true $\Pi_{2}$-sentence $\pi$ such that EA $+\pi$ is not closed under $\Delta_{1}$-IR. The aim of this section is to establish the following result. 
Theorem 2. For any $\Pi_{3}$-sentence $\pi, I \Delta_{1}+\pi$ is $\Pi_{2}$-conservative over $\pi+\Delta_{1}$-IR.

Proof. To prove this theorem we first formulate a certain Gentzen-like rule corresponding to $I \Delta_{1}$ and by free cut-elimination procedure establish the $\Pi_{2^{-}}$ conservativity of this rule over its restricted version. Then the restricted version is shown to be equivalent to $\Delta_{1}$-IR.

We use the standard formalism of Tait calculus [11], that is, we treat sequents as sets of formulas (understood as their disjunctions). Formulas are built up by connectives $\wedge, \vee$ and quantifiers from atomic formulas and their negations (hence, $\neg, \rightarrow, \leftrightarrow$ are defined operations). We also keep apart the alphabets of free and bound variables. We shall work in the language of EA.

Our sequential version of $I \Delta_{1}$ is the following rule:

$$
\Delta_{1}-\mathrm{IR}^{\prime}: \quad \frac{\Delta, \neg \varphi(a), \neg \psi(a) \quad \Delta, \varphi(a), \psi(a) \quad \Delta, \varphi(0) \quad \Delta, \neg \varphi(a), \varphi(a+1)}{\Delta, \varphi(t)}
$$

where $t$ is a term, $\varphi$ and $\psi$ are $\Sigma_{1}$-formulas, $\Delta$ is a finite set of formulas, and the free variable $a$ does not occur in $\Delta, \varphi(t) . \varphi(t)$ is called the main formula of the inference.

It is clear that $I \Delta_{1}$ is closed under $\Delta_{1}-\mathrm{IR}^{\prime}$, if one translates the sequents as disjunctions. Using $\Delta_{1}-\mathrm{IR}^{\prime}$ it is also easy to derive the schema $I \Delta_{1}$, if one takes for $\Delta$ the set $\{\neg \forall x(\varphi(x) \leftrightarrow \psi(x)), \neg \varphi(0), \neg \forall x(\varphi(x) \rightarrow \varphi(x+1))\}$ and for $t$ a free variable. ${ }^{3}$

Then we obtain the following version of free cut-elimination.

Lemma 10. Every derivation using $\Delta_{1}-\mathrm{IR}^{\prime}$ and predicate logic can be effectively transformed into a derivation in which every cut-formula or its negation is the main formula of an induction inference.

Proof. This can be shown essentially as in [11]. We omit the details.

Let $\Delta_{1}-\mathrm{IR}^{\prime \prime}$ denote the rule $\Delta_{1}-\mathrm{IR}^{\prime}$ with the restriction that $\Delta$ consists of purely universal or purely existential formulas. As a corollary of the free cutelimination we observe the following lemma.

Lemma 11. Let $T$ be a $\Pi_{3}$-axiomatized theory containing $E A$. Then $T+I \Delta_{1}$ is $\Pi_{2}$-conservative over $T+\Delta_{1}-\mathrm{IR}^{\prime \prime}$.

Proof. We may assume that the axioms of $T$ are brought into prenex normal form, that is, they have the form

$$
\forall x_{1} \ldots \forall x_{k} \exists y_{1} \ldots \exists y_{l} A\left(x_{1}, \ldots, x_{k}, y_{1}, \ldots, y_{l}\right),
$$

where $A$ is a $\Pi_{1}$-formula. Consider a derivation in predicate logic together with $\Delta_{1}-\mathrm{IR}^{\prime}$ of a sequent $\neg T, \Sigma$, where $\neg T$ denotes a finite number of negated axioms of $T$ as above, and $\Sigma$ is a set of $\Sigma_{1}$-formulas (possibly, with parameters). By

${ }^{3}$ Later we shall treat the other nonlogical axioms of $I \Delta_{1}$, including equality axioms, as side assumptions. 
free cut-elimination we may assume that every cut-formula in this derivation is the main formula of a $\Delta_{1}$-induction inference. Thus, it has complexity $\Sigma_{1}$ or $\Pi_{1}$. Every formula occurring in the derivation is a subformula of a cut-formula or of a formula occurring in the end sequent. Thus, it has to be $\Sigma_{1}$ or $\Pi_{1}$ unless it belongs to $\neg T$ or has one of the following forms:

1. $\exists x_{m+1} \ldots \exists x_{k} \forall y_{1} \ldots \forall y_{l} \neg A\left(t_{1}, \ldots, t_{m}, x_{m+1}, \ldots, x_{k}, y_{1}, \ldots, y_{l}\right)$, where $t_{i}$ are terms, $1 \leq m \leq k$;

2. $\forall y_{m+1} \ldots \forall y_{l} \neg A\left(t_{1}, \ldots, t_{k}, a_{1}, \ldots, a_{m}, y_{m+1}, \ldots, y_{l}\right)$, where $t_{i}$ are terms, $a_{j}$ are distinct free variables, $1 \leq m<l$.

Now consider any application of $\Delta_{1}-\mathrm{IR}^{\prime}$ in this derivation. We may apply the quantifier rules to all the side formulas of its premises which have types 2 and 1 in order to replace such occurrences by the negated instances of the axioms of $T$. Thus, we obtain the sequents in which all formulas either belong to $\neg T$ or to $\Sigma_{1} \cup \Pi_{1}$. The former can then be cutted away by axiom sequents

$$
\forall x_{1} \ldots \forall x_{k} \exists y_{1} \ldots \exists y_{l} A\left(x_{1}, \ldots, x_{k}, y_{1}, \ldots, y_{l}\right)
$$

In this way every application of $\Delta_{1}$-IR' is transformed into the one with $\Sigma_{1} \cup \Pi_{1}$ side formulas, that is, into an application of $\Delta_{1}-\mathrm{IR}^{\prime \prime}$. The whole derivation is then transformed into a derivation in $T+\Delta_{1}-\mathrm{IR}^{\prime \prime}$.

Notice that the rule $\Delta_{1}$-IR" maps $\Pi_{2}$-formulas to $\Pi_{2}$-formulas. We would like to simplify it a bit. Notice that by introducing fresh free variables for the universal quantifiers we may assume all the side formulas in an application of $\Delta_{1}-\mathrm{IR}^{\prime \prime}$ to be $\Sigma_{1}$. Then we obtain the following Hilbert-style form of this rule:

$$
\frac{A \rightarrow \forall x(\varphi(x) \leftrightarrow \psi(x))}{A \rightarrow I_{\varphi}}
$$

where $\varphi \in \Sigma_{1}$ and $A, \psi \in \Pi_{1}$ (A, $\varphi$ and $\psi$ may also contain parameters).

Lemma 12. The rules $\Delta_{1}-\mathrm{IR}^{\prime \prime}$ and $(*)$ are equivalent.

Proof. To derive $\Delta_{1}-\mathrm{IR}^{\prime \prime}$ from $(*)$ accumulate all the side formulas $\Delta$ into the formula $A$. For the opposite reduction, let $\Delta$ be the set $\{\neg A, \psi(0), \exists x(\varphi(x) \wedge$ $\psi(x+1))\}$.

For a rule $R$, let $[T, R]$ denote the closure of a theory $T$ under the unnested applications of $R$. Rules $R_{1}$ and $R_{2}$ are congruent if, for any $T$ extending EA, $\left[T, R_{1}\right] \equiv\left[T, R_{2}\right]$ (see [1]). The proof of the previous lemma actually shows that $\Delta_{1}-\mathrm{IR}^{\prime \prime}$ and $(*)$ are congruent. Obviously, congruence implies deductive equivalence (interderivability) of the rules.

Lemma 13. $\Delta_{1}-\mathrm{IR}^{\prime \prime}$ and $\Delta_{1}-\mathrm{IR}$ are congruent. 
Proof. It is sufficient to show that $\left[T, \Delta_{1}-\mathrm{IR}^{\prime \prime}\right] \subseteq\left[T, \Delta_{1}-\mathrm{IR}\right]$ for any theory $T$ extending EA. We also do not distinguish between $\Delta_{1}-\mathrm{IR}^{\prime \prime}$ and the rule $(*)$. Assume

$$
T \vdash A(a) \rightarrow \forall x(\varphi(x, a) \leftrightarrow \psi(x, a)),
$$

where $a$ w.l.o.g. is the only parameter in the given formulas. Given $\Sigma_{1}$-formulas $\sigma=\exists u \sigma_{0}(u)$ and $\tau=\exists u \tau_{0}(u)$ with $\sigma_{0}$ and $\tau_{0}$ bounded, we shall use the following witness comparison notation:

$$
\begin{aligned}
& \sigma \prec \tau:=\exists u\left(\sigma_{0}(u) \wedge \forall y \leq u \neg \tau_{0}(y)\right), \\
& \sigma \preceq \tau:=\exists u\left(\sigma_{0}(u) \wedge \forall y<u \neg \tau_{0}(y)\right) .
\end{aligned}
$$

We shall use the same notation for formulas logically equivalent to $\Sigma_{1}$, having in mind some fixed way of bringing them into graphically $\Sigma_{1}$-form (no matter which). The following properties hold provably in EA:

$$
\begin{aligned}
& (\sigma \vee \tau) \rightarrow \sigma \preceq \tau \vee \tau \prec \sigma, \\
& \sigma \preceq \tau \rightarrow \sigma, \\
& \neg(\sigma \preceq \tau \wedge \tau \prec \sigma), \\
& \sigma \wedge \neg \tau \rightarrow \sigma \prec \tau .
\end{aligned}
$$

Now we define

$$
\begin{aligned}
& B_{0}(a, x):=[\neg A \preceq(\varphi \vee \neg \psi)] \vee(\varphi \preceq \neg \psi), \\
& B_{1}(a, x):=[(\varphi \vee \neg \psi) \prec \neg A] \wedge(\neg \psi \prec \varphi) .
\end{aligned}
$$

Notice that both formulas are $\Sigma_{1}$. We claim that

$$
T \vdash \forall a, x\left(B_{0}(a, x) \leftrightarrow \neg B_{1}(a, x)\right) .
$$

The implication $(\rightarrow)$ follows from (4). For a proof of $(\leftarrow)$ by $(1)$ and $(2)$ we obtain

$$
T \vdash[\neg A \preceq(\varphi \vee \neg \psi)] \vee[(\varphi \vee \neg \psi) \prec \neg A] .
$$

Therefore,

$$
T \vdash \neg[(\varphi \vee \neg \psi) \prec \neg A] \rightarrow B_{0}
$$

On the other hand,

$$
\begin{aligned}
T \vdash(\varphi \vee \neg \psi) \prec \neg A & \rightarrow(\varphi \vee \neg \psi) \\
& \rightarrow(\varphi \preceq \neg \psi) \vee(\neg \psi \prec \varphi),
\end{aligned}
$$

and thus

$$
\begin{aligned}
T \vdash(\varphi \vee \neg \psi) \prec \neg A \wedge \neg(\neg \psi \prec \varphi) & \rightarrow \varphi \preceq \neg \psi \\
& \rightarrow B_{0} .
\end{aligned}
$$


It follows that

$$
T \vdash \neg B_{1}(a, x) \rightarrow B_{0}(a, x) .
$$

We have shown that $\Delta_{1}$-IR is applicable to $B_{0}$ and therefore

$$
\left[T, \Delta_{1}-\mathrm{IR}\right] \vdash I_{B_{0}}(a) .
$$

On the other hand, by (3)

$$
\begin{aligned}
T \vdash A & \rightarrow \neg[\neg A \preceq(\varphi \vee \neg \psi)] \\
& \rightarrow\left(B_{0} \leftrightarrow \varphi \preceq \neg \psi\right) .
\end{aligned}
$$

By (1) and (5) we obtain

$$
T \vdash A \rightarrow(\varphi \preceq \neg \psi \leftrightarrow \varphi),
$$

and since $x$ does not occur in $A$ this implies

$$
\begin{aligned}
T \vdash A(a) & \rightarrow \forall x\left(B_{0}(a, x) \leftrightarrow \varphi(x, a)\right) \\
& \rightarrow\left(I_{B_{0}}(a) \leftrightarrow I_{\varphi}(a)\right) .
\end{aligned}
$$

Hence,

$$
T \vdash I_{B_{0}}(a) \rightarrow\left(A(a) \rightarrow I_{\varphi}(a)\right),
$$

and

$$
\left[T, \Delta_{1}-\mathrm{IR}\right] \vdash A(a) \rightarrow I_{\varphi}(a) .
$$

This completes the proof of Lemma and of Theorem 2.

\section{More on parameter restrictions}

Here we introduce a version of $I \Delta_{1}$ with a natural parameter restriction, which yields an axiomatization of the set of $\Sigma_{3}$-consequences of $I \Delta_{1}$.

Consider the following schema:

$$
s I \Delta_{1}: \quad \forall x, \boldsymbol{z}(\varphi(x, \boldsymbol{z}) \leftrightarrow \psi(x, \boldsymbol{z})) \rightarrow \forall \boldsymbol{z} I_{\varphi}(\boldsymbol{z}),
$$

Here $\varphi \in \Sigma_{1}, \psi \in \Pi_{1}$, and $\boldsymbol{z}$ denotes all the parameters in $\varphi$ and $\psi . s I \Delta_{1}$ can be called $\Delta_{1}$-induction schema with separated parameters, in the sense that the parameters $\boldsymbol{z}$ are quantified apart in the premise and in the conclusion.

First, we are going to show that $s I \Delta_{1}$ is equivalent to $S \Delta_{1}^{-}$. To this end, we analyze the corresponding inference rules. $\Delta_{1}$-search rule is as follows:

$$
\Delta_{1} \text {-SR : } \quad \frac{\forall x(\varphi(x) \leftrightarrow \psi(x))}{S_{\varphi}}
$$

where $\varphi \in \Sigma_{1}$ and $\psi \in \Pi_{1}$. It is clear that $\Delta_{1}$-SR is congruent to $\Delta_{1}$-IR. We let $\Delta_{1}-\mathrm{SR}^{-}$denote the rule $\Delta_{1}$-SR for formulas $\varphi(x)$ and $\psi(x)$ without extra parameters. 
Lemma 14. $\Delta_{1}-\mathrm{SR}^{-}$is congruent to $\Delta_{1}-\mathrm{IR}$.

Proof. It is sufficient to derive $\Delta_{1}$-SR by a single application of $\Delta_{1}-\mathrm{SR}^{-}$. So, consider an arbitrary theory $T$ and assume

$$
T \vdash \forall z, x(\varphi(x, z) \leftrightarrow \psi(x, z))
$$

(w.l.o.g. we consider a single parameter $z$ ). We have to show that

$$
\left[T, \Delta_{1}-\mathrm{SR}^{-}\right] \vdash \forall z S_{\varphi}(z) .
$$

We shall reason informally and treat $\varphi$ as a $0-1$-valued total computable function $f(x, z)$ (formally, $f(x, z)=y$ will stand for $(\varphi(x, z) \wedge y=0) \vee(\neg \psi(x, z) \wedge y=1)$ ).

We have to prove in $\left[T, \Delta_{1}-\mathrm{SR}^{-}\right]$:

$$
\forall z, a(f(0, z)=0 \wedge f(a, z)=1 \rightarrow \exists x<a(f(x, z)=0 \wedge f(x+1, z)=1)) .
$$

The problem is to get rid of the parameter $z$.

Consider the elementary function

$$
h(z, a):=\sum_{i<\langle z, a\rangle}\left((i)_{1}+1\right)
$$

where $(i)_{1}$ denotes the second component of the pair $i$ in the standard one-to-one encoding of pairs. We define a new function $g$ by putting together pieces of $f$ as follows:

$$
g(h(z, a)+x):=f(x, z), \quad \text { for all } x \leq a .
$$

In other words, $g$ restricted to the interval $[h(z, a), h(z, a)+a]$ encodes the first $a+1$ values of $f(\cdot, z)$. The definition of $h$ ensures that for different pairs $\langle z, a\rangle$ these intervals do not intersect and cover $\mathbb{N}$, that is, $g$ is well-defined. (In fact, the intervals are ordered in the order of pairs $\langle z, a\rangle$.)

It is also clear from our assumption about $f$ that $g$ is total and recursive, hence, definable by a $\Delta_{1}$-formula in $T$. So, if $f(0, z)=0$ and $f(a, z)=1$, by $\Delta_{1}$ SR $^{-}$applied to $g$ and the interval $[h(z, a), h(z, a)+a]$ we obtain $g(h(z, a))=0$ and $g(h(z, a)+a)=1$, hence

$$
\exists x \in[h(z, a), h(z, a)+a)(g(x)=0 \wedge g(x+1)=1) .
$$

This implies

$$
\exists x<a(f(x, z)=0 \wedge f(x+1, z)=1))
$$

by the definition of $g$.

Corollary 5. $s I \Delta_{1} \equiv S \Delta_{1}^{-}$.

Proof. Clearly, $S \Delta_{1}^{-} \subseteq s I \Delta_{1}$, because $I_{\neg \varphi(x+a)} \Rightarrow S_{\varphi}$. For the opposite inclusion let $\pi$ denote the formula $\forall \boldsymbol{z}, x(\varphi(x, \boldsymbol{z}) \leftrightarrow \psi(x, \boldsymbol{z}))$ and reason as follows:

$$
\begin{aligned}
S \Delta_{1}^{-}+\pi & \supseteq\left[\pi, \Delta_{1}-\mathrm{SR}^{-}\right] \\
& \supseteq\left[\pi, \Delta_{1}-\mathrm{IR}\right] \\
& \vdash I_{\varphi} .
\end{aligned}
$$


Since $S \Delta_{1}^{-}$is a schema and $\pi$ is closed, Deduction theorem yields

$$
S \Delta_{1}^{-} \vdash \forall x, \boldsymbol{z}(\varphi(x, \boldsymbol{z}) \leftrightarrow \psi(x, \boldsymbol{z})) \rightarrow \forall \boldsymbol{z} I_{\varphi}(\boldsymbol{z}) .
$$

So, we have the following inclusions:

$$
\mathrm{EA} \subseteq I \Delta_{1}^{-} \subset S \Delta_{1}^{-} \equiv s I \Delta_{1} \subset I \Delta_{1} .
$$

Whether $I \Delta_{1}^{-}$properly extends EA is an open question (we conjecture that it does). Notice that $s I \Delta_{1} \nvdash I \Delta_{1}$, because $S \Delta_{1}^{-}$follows from $T h_{\Pi_{2}}(\mathbb{N})$, whereas $I \Delta_{1}$ does not, by Theorem 1 .

Now we prove that $s I \Delta_{1}$ (or $S \Delta_{1}^{-}$) precisely axiomatizes the $\Sigma_{3}$-consequences of $I \Delta_{1}$. This is interesting, because $S \Delta_{1}^{-}$is a set of boolean combinations of $\Pi_{2^{-}}$ sentences. Thus, the relation of $I \Delta_{1}$ to $S \Delta_{1}^{-}$is the same as that of $I \Sigma_{1}$ to $I \Sigma_{1}^{-}$ and of $B \Sigma_{1}$ to $B \Sigma_{1}^{-}$[6]. In the present situation, though, the parameter-free schema $I \Delta_{1}^{-}$is too weak. So, in our opinion, $S \Delta_{1}^{-}$is a more adequate formulation of parameter-free $\Delta_{1}$-induction than $I \Delta_{1}^{-}$.

Corollary 6. I $\Delta_{1}$ is conservative over sI $\Delta_{1}$ (and $S \Delta_{1}^{-}$) for $\Sigma_{3}$-sentences.

Proof. Assume $I \Delta_{1} \vdash \sigma$, where $\sigma$ is a $\Sigma_{3}$-sentence. Then $\neg \sigma+I \Delta_{1} \vdash \perp$, whence by Lemmas 11 and 13

$$
\neg \sigma+\Delta_{1}-\mathrm{IR} \vdash \perp .
$$

Notice that $s I \Delta_{1}$ is closed under $\Delta_{1}$-IR, so by Lemma 12

$$
\neg \sigma+s I \Delta_{1} \vdash \perp \text {. }
$$

Hence, by Deduction theorem, $s I \Delta_{1} \vdash \sigma$.

\section{On $I \Delta_{1}$ versus $B \Sigma_{1}$ problem}

In this section we characterize the classes of provably total computable functions of extensions of $I \Delta_{1}$ by true $\Pi_{2}$-sentences. Thereby a reduction of the question of separation of $I \Delta_{1}$ from $B \Sigma_{1}$ to a purely computation-theoretic question is obtained.

Our approach to the separation problem is similar to the one in Section 3. If one constructs a true $\Pi_{2}$-sentence $\pi$ such that

$$
\mathcal{F}\left(\pi+I \Delta_{1}\right) \neq \mathcal{F}\left(\pi+B \Sigma_{1}\right),
$$

then $I \Delta_{1} \not \equiv B \Sigma_{1}$. By the results of [2] we have a simple characterization of the class $\mathcal{F}\left(\pi+B \Sigma_{1}\right)$.

Lemma 15. Let $\pi$ be a true $\Pi_{2}$-sentence of the form $\forall x \exists y \varphi(x, y)$, with $\varphi$ bounded. Then $\mathcal{F}\left(\pi+B \Sigma_{1}\right)=\mathbf{E}(f)$, where $f(x)=\mu y \cdot \varphi(x, y)$. 
It is known that $\mathbf{E}(f)=\mathbf{C}(\bar{f})$, where $\bar{f}(x):=\langle f(0), \ldots, f(x)\rangle$. Moreover, if $f$ has an elementary graph, then $\mathbf{E}(f)=\mathbf{C}\left(\max _{i \leq x} f(i)\right)$. So, any of the functions $\bar{f}(x)$ or $\max _{i \leq x} f(i)$ can be considered as the 'hardest' computation problem in the class $\mathbf{E}(f)$.

Corollary 7. Let $\pi$ be a true $\Pi_{2}$-sentence of the form $\forall x \exists y \varphi(x, y)$, with $\varphi$ bounded, and let $f(x)=\mu y . \varphi(x, y)$. If $\max _{i \leq x} f(i) \notin \mathcal{F}\left(\pi+\Delta_{1}\right.$-IR $)$, then I $\Delta_{1} \nvdash$ $B \Sigma_{1}$.

Proof. It only has to be noticed that Theorem 2 implies that $\mathcal{F}\left(\pi+\Delta_{1}-\mathrm{IR}\right)=$ $\mathcal{F}\left(\pi+I \Delta_{1}\right)$. That $\pi+B \Sigma_{1}$ proves the totality of $\max _{i \leq x} f(i)$ is obvious.

Our next goal is to obtain a recursion-theoretic characterization of the class $\mathcal{F}\left(\pi+\Delta_{1}\right.$-IR $)$. In order to analyze $\Delta_{1}$-IR in terms of provably recursive functions we introduce two equivalent forms of this rule. Recall that by Lemma $14 \Delta_{1}$-IR is congruent to the rule $\Delta_{1}-\mathrm{SR}^{-}$.

Lemma 16. $\Delta_{1}-\mathrm{SR}^{-}$is congruent to the following rule:

$$
\frac{\forall x \exists y \leq 1 \Phi_{\bar{e}}(x) \simeq y}{\forall a<b\left(\Phi_{\bar{e}}(a) \simeq 1 \vee \Phi_{\bar{e}}(b) \simeq 0 \vee \exists x \in[a, b)\left(\Phi_{\bar{e}}(x) \simeq 0 \wedge \Phi_{\bar{e}}(x+1) \simeq 1\right)\right)}
$$

Proof. Both reductions are straightforward modulo the standard characterization of the class of recursive predicates.

Our second version looks more complicated, but it has a technical advantage of having quantifiers explicit. Let $J(g, a, b, z)$ be the following formula (with a function parameter $g$ ):

$$
\begin{array}{rl}
\exists x, u, v \leq z & z=\langle x, u, v\rangle \wedge \\
& {\left[\left(a \leq x<b \wedge(u)_{0}=0 \wedge(v)_{0}=1 \wedge g(x)=u \wedge g(x+1)=v\right) \vee\right.} \\
& \left(x=a \wedge(u)_{0}=1 \wedge v=0 \wedge g(a)=u\right) \vee \\
& \left.\left(x=b \wedge u=0 \wedge(v)_{0}=0 \wedge g(b)=v\right)\right]
\end{array}
$$

$J\left(\Phi_{e}, a, b, z\right)$ denotes the result of replacing in $J$ atomic subformulas of the form $g(x)=y$ by $\Phi_{e}(x) \simeq y$.

Lemma 17. $\Delta_{1}-\mathrm{SR}^{-}$is congruent to

$$
\frac{\forall x \exists y \Phi_{\bar{e}}(x) \simeq y \quad \forall x, y\left(\Phi_{\bar{e}}(x) \simeq y \rightarrow(y)_{0} \leq 1\right)}{\forall a<b \exists z J\left(\Phi_{\bar{e}}, a, b, z\right)}
$$

Proof. To reduce $\Delta_{1}-\mathrm{SR}^{-}$to this rule use Lemma 16 and go from a computable 0-1-valued function $\Phi_{e}$ to the function $\Phi_{e^{\prime}}$ which, if converges, outputs the pair $\left\langle\Phi_{e}(x), z\right\rangle$, where $z$ is the full protocol of the computation of $\Phi_{e}(x)$. (Notice that the graph of $\Phi_{e^{\prime}}$ is elementary.)

For the opposite reduction go from $\Phi_{e}(x)$ to the function $\left(\Phi_{e}(x)\right)_{0}$. 
In the following we shall not distinguish between $\Delta_{1}-\mathrm{SR}^{-}$and any of the rules in Lemmas 16, 17.

We introduce a new subrecursive search operator, which corresponds to this rule. The status of the operator is similar to that of the bounded $\mu$-operator or primitive recursion in that it allows to construct new total computable functions from the old ones. It corresponds to the interval search principle in the same way as the bounded $\mu$-operator corresponds to the least element principle.

Assume $g$ is a function such that, for all $x,(g(x))_{0} \leq 1$. Then $S_{g}$ denotes the function

$$
S_{g}(a, b):=\mu z . J(g, a, b, z) .
$$

If $g$ is computable, then so is $S_{g}$. Intuitively, $S_{g}(a, b)$ tries to compute all the values of $g$ on interval $[a, b]$ in parallel. One eventually finds a point $x \in[a, b)$ such that $(g(x))_{0}=0$ and $(g(x+1))_{0}=1$, or one establishes that $(g(a))_{0}=1$ or $(g(b))_{0}=0$. Then one outputs such an $x$ together with a witness $z$ that $x$ is as required.

$\mathbf{S}(K)$ will denote the closure of $K \cup \mathcal{E}$ under composition and the search operator $g \mapsto S_{g}$. We have the following natural characterization.

Theorem 3. Let $\pi$ be a true $\Pi_{2}$-sentence of the form $\forall x \exists y \varphi(x, y)$, with $\varphi$ bounded. Then $\mathcal{F}\left(\pi+I \Delta_{1}\right)=\mathbf{S}(f)$, where $f(x)=\mu y \cdot \varphi(x, y)$.

Proof. The class of provably total computable functions only depends on the $\Pi_{2}$-fragment of a theory, hence by Theorem 2 and Lemma 14

$$
\mathcal{F}\left(\pi+I \Delta_{1}\right)=\mathcal{F}\left(\pi+\Delta_{1}-\mathrm{IR}\right)=\mathcal{F}\left(\pi+\Delta_{1} \mathrm{SR}^{-}\right) .
$$

It is also clear from Lemma 17 that $\mathcal{F}\left(\pi+\Delta_{1}\right.$ SR $\left.^{-}\right)$is closed under the search operator, so $\mathbf{S}(f) \subseteq \mathcal{F}\left(\pi+\Delta_{1} \mathrm{SR}^{-}\right)$.

For the converse inclusion we show by induction on the depth of nestings of applications of $\Delta_{1}$ SR $^{-}$that every $g \in \mathcal{F}\left(\pi+\Delta_{1}-\mathrm{SR}^{-}\right)$belongs to $\mathbf{S}(f)$.

Indeed, let $T$ be the extension of EA $+\pi$ obtained by $\leq n$ nested applications of $\Delta_{1}$-SR ${ }^{-}$. Then $T$ is $\Pi_{2}$-axiomatized, and by the induction hypothesis $\mathcal{F}(T) \subseteq$ $\mathbf{S}(f) .\left[T, \Delta_{1}-\mathrm{SR}^{-}\right]$is axiomatizable over $T$ by $\Pi_{2}$-sentences of the form

$$
\forall b \forall a<b \exists z J\left(\Phi_{\bar{e}}, a, b, z\right)
$$

such that

$$
\forall x \exists y \Phi_{\bar{e}}(x) \simeq y \wedge \forall x, y\left(\Phi_{\bar{e}}(x) \simeq y \rightarrow(y)_{0} \leq 1\right)
$$

is provable in $T$. Moreover, we may only consider the functions $\Phi_{e}$ with elementary graphs (as in the proof of Lemma 17). Then the witnessing functions for the formulas (6) are precisely the functions $S_{\Phi_{e}}(a, b)$, for all such $\Phi_{e}$. Hence, by Lemma 2 and the induction hypothesis

$$
\begin{aligned}
\mathcal{F}\left(\left[T, \Delta_{1}-\mathrm{SR}^{-}\right]\right) & \subseteq \mathbf{C}\left(\mathcal{F}(T) \cup\left\{S_{g}: g \in \mathcal{F}(T)\right\}\right) \\
& \subseteq \mathbf{S}(f) .
\end{aligned}
$$

This proves the induction step. 
From Corollary 7 we obtain

Corollary 8. If there is a total recursive function $f$ with an elementary graph such that $\max _{i \leq x} f(i) \notin \mathbf{S}(f)$, then $I \Delta_{1} \not \equiv B \Sigma_{1}$.

The meaning of this corollary for us is that it reduces the original separation problem to the question of constructing a suitable function $f$. (Notice that the assumption of the corollary is equivalent to the problem of separating the classes $\mathbf{S}(f)$ and $\mathbf{E}(f)$ by a function $f$ with an elementary graph.) Formally, our reduction is one-sided, that is, only a sufficient condition for the separation is suggested (e.g., information is being lost when we go from theories to the corresponding classes of functions). However, we conjecture that the sufficient condition actually holds and can be established using some existent techniques. Moreover, we also believe that any explicit representation of $\max _{i<x} f(i)$ as an $\mathbf{S}(f)$-function (unlikely though it may be) would, in practice, yield a proof of $B \Sigma_{1}$ in $I \Delta_{1}$.

At present we only have partial results in the direction of separating $\mathbf{S}(f)$ and $\mathbf{E}(f)$. The best we know is that $S$-operators without nestings do not suffice to generate $\mathbf{E}(f)$ for a suitable recursive function $f$. But the same also holds for the stronger bounded $\mu$-operators without nestings, by [3]. Doubly nested $\mu$ operators already suffice to generate $\mathbf{E}(f)$, so this result does not really indicate a difference between $\mathbf{S}(f)$ and $\mathbf{E}(f)$.

\section{Concluding remarks}

Apart from the intriguing $I \Delta_{1}$ versus $B \Sigma_{1}$ problem some interesting particular questions concerning $I \Delta_{1}$ are left open. Here we list some of them:

1. Is there a total function $f$ with an elementary graph such that $\max _{i \leq x} f(i) \notin$ $\mathbf{S}(f)$ ? Notice that the existence of such a function implies a stronger kind of separation between $I \Delta_{1}$ and $B \Sigma_{1}$, that is, an answer to the following question:

2. Does $B \Sigma_{1}$ follow from $I \Delta_{1}$ together with all true $\Pi_{2}$-sentences?

3. Is there a natural description of the class $\mathbf{S}(f)$ in terms of oracle Turing machines? (We have in mind devices like, e.g., nondeterministic bounded query Turing machines.)

4. Does $I \Delta_{1}^{-}$follow from EA? From any r.e. set of true $\Pi_{1}$-sentences?

5. Prove the analogs of the results of this paper for schemata of higher logical complexity, in particular, for $I \Delta_{n}$ where $n>1$. (This seems to be routine using, e.g., the techniques of $[1,2]$.)

The results of this paper also strengthen the links between fragments of arithmetic and query complexity.

\section{Acknowledgements}

This paper was written during my stay in 1999-2000 at the University of Technology in Vienna and was supported in part by Lise Meitner research fellowship 
of the Austrian Funds for Science and Research (FWF). I thank Matthias Baaz

for many stimulating discussions.

\section{References}

1. L.D. Beklemishev. Induction rules, reflection principles, and provably recursive functions. Annals of Pure and Applied Logic, 85:193-242, 1997.

2. L.D. Beklemishev. A proof-theoretic analysis of collection. Archive for Mathematical Logic, 37:275-296, 1998.

3. L.D. Beklemishev. Open least element principle and bounded query computation. In J. Flum and M. Rodrigues-Artalejo, editors, Lecture Notes in Computer Science 1683. Computer Science Logic, 13-th international workshop, CSL'99. Madrid, Spain, September 20-25, 1999. Proceedings, page ??? Springer-Verlag, Berlin, 1999.

4. P. Clote and J. Krajíček. Open problems. In P. Clote and J. Krajíček, editors, Arithmetic, Proof Theory, and Computational Complexity, pages 1-19. Oxford University Press, Oxford, 1993.

5. P. Hájek and P. Pudlák. Metamathematics of First Order Arithmetic. SpringerVerlag, Berlin, Heidelberg, New York, 1993.

6. R. Kaye, J. Paris, and C. Dimitracopoulos. On parameter free induction schemas. Journal of Symbolic Logic, 53(4):1082-1097, 1988.

7. J. Paris. A hierarchy of cuts in models of arithmetic. In Model theory of algebra and arithmetic. Proceedings, Karapascz, Poland, 1979. Lecture Notes in Mathematics, v. 834, pages 312-337. Springer-Verlag, 1980.

8. C. Parsons. On a number-theoretic choice schema and its relation to induction. In A. Kino, J. Myhill, and R.E. Vessley, editors, Intuitionism and Proof Theory, pages 459-473. North Holland, Amsterdam, 1970.

9. C. Parsons. On n-quantifier induction. Journal of Symbolic Logic, 37(3):466-482, 1972.

10. A. Rastsvetaev and L. Beklemishev. On the query complexity of finding a local maximum point. Manuscript, 6 pages, 2000.

11. H. Schwichtenberg. Some applications of cut-elimination. In J. Barwise, editor, Handbook of Mathematical Logic, pages 867-896. North Holland, Amsterdam, 1977. 